\begin{tabular}{lllllllllll} 
O P E R A T I O N S R E S E A R C H A N D D E C I S I O N S \\
\hline No. 1
\end{tabular}

DOI: $10.37190 /$ ord210105

\title{
DETECTING CONGESTION IN DEA BY SOLVING ONE MODEL
}

\author{
MARYAM SHADAB $^{1}$, SABER SAATI $^{1 *}$ \\ REZA FARZIPOOR SAEN ${ }^{2}$, MOHAMMAD KHOVEYNI ${ }^{3}$, AMIN MOSTAFAEE $^{1}$ \\ ${ }^{1}$ Department of Mathematics, North Tehran Branch, Islamic Azad University, Tehran, Iran \\ ${ }^{2}$ Faculty of Business, Sohar University, Sohar, Oman \\ ${ }^{3}$ Department of Applied Mathematics, Yadegar-e-Imam Khomeini (RAH) Shahre Rey Branch, \\ Islamic Azad University, Tehran, Iran
}

\begin{abstract}
The presence of input congestion is one of the key issues that result in lower efficiency and performance in decision-making units $(D M U \mathrm{~s})$. So, determination of congestion is of prime importance, and removing it improves the performance of $D M U$ s. One of the most appropriate methods for detecting congestion is Data Envelopment Analysis (DEA). Since the output of inefficient units can be increased by keeping the input constant through projecting on the weak efficiency frontier, it is unnecessary to determine the congested inefficient $D M U$ s. Therefore, in this case, we solely determine congested vertex units. Towards this aim, only one LP model in DEA is proposed and the status of congestion (strong congestion and weak congestion) obtained. In our method, a vertex unit under evaluation is eliminated from the production technology, and then, if there exists an activity that belongs to the production technology with lower inputs and higher outputs compared with the omitted unit, we say vertex unit evidences congestion. One of the features of our model is that by solving only one LP model and with easier and fewer calculations compared to other methods, congested units can be identified. Data set obtained from Japanese chain stores for a period of 27 years is used to demonstrate the applicability of the proposed model and the results are compared with some previous methods.
\end{abstract}

Keywords: data envelopment analysis, vertex units, congestion

\section{Introduction}

Evaluation of decision-making units (DMUs) performance and efficiency is a significant topic for many researchers and scholars. One of the most efficient methods for assessing the relative efficiency of peer $D M U$ s based on LP is data envelopment analysis

*Corresponding author, email address: s_saatim@iau-tnb.ac.ir Received 28 July 2020, accepted 1March 2021 
(DEA). DEA is a non-parametric method used to assess homogenous $D M U$ s that consume multiple inputs to produce multiple outputs. The two basic DEA models are CCR model proposed by Charnes et al. [1], and BCC model proposed by Banker et al. [2], which rely on constant returns to scale (CRS) and variable returns to scale (VRS) assumption, respectively, to obtain relative efficiency of $D M U$ s.

The existence of congestion in inputs is one of the factors that decrement the efficiency of $D M U$ s. Congestion is an event in the production processes in which the increment in one or more inputs does not increase outputs. According to the definition, congestion can be considered as a shortage in outputs. Eliminating congestion leads to improved performance and efficiency of $D M U$ s. A real sample of congestion is coal mine where the miners are working in narrow crowded shafts and pits, the amount of minerals extracted will be reduced [3].

Many studies have been carried out to evaluate congestion in DEA context. The study of congestion was started by Färe and Svensson [4]. Their study was later developed by Färe and Grosskopf [5] who presented a model based on DEA for detecting congested DMUs.

Färe et al. [6] suggested two DEA models in input-oriented and output-oriented (called FGL approach) for identifying congested units, but their method could not compute its value. The detecting congestion issue was seriously investigated by Cooper et al. [7]. They played a major role in determining DEA-based congestion: for instance, they proposed a slack-based approach (called CTT model), which evaluated the congested units and calculated the amount of congestion in each input. Bracket et al. [8] (called BCSW model) extended CTT model to examine the tradeoff between employment and output. Cooper et al. [9] integrated the two stages of CTT approach into a single model to determine congestion. Next, Jahanshahloo and Khodabakhshi [10] suggested one LP model for determining input congestion based on relax input combination for improving outputs. Next, Khodabakhshi [11] explored the input congestion in stochastic DEA. Also, Wei and Yan [12] investigated the necessary and sufficient conditions for the occurrence of congestion according to the type of returns to scale (RTS) due to DEA efficiency. Also, Tone and Sahoo [13] defined a new production possibility set (PPS) with the assumption of convexity and strong output disposability and omitting input possibility postulate named $T_{\text {convex. }}$. They introduced the new concepts of "strong congestion" and "weak congestion" and proposed to identify the congested $D M U$ s.

Some previous studies deal with the problem of determining congested inefficient units. According to these studies, the congested inefficient unit is defined at its efficient projection, which is determined via the congestion-based DEA model. If multiple projections occur, one of them is chosen arbitrarily, whereas choosing any of the efficient projections leads to changes in their congestion status. To overcome this problem, further studies have been conducted on this crucial issue. For instance, Sueyoshi and Sekitiani [14] propose the definition of "wide congestion" which covers both strong and weak congestion and suggest an approach that produces a unique optimal solution and 
a unique projection for inefficient units. Then, two DEA models are proposed which could uniquely determine the status of wide congestion. Mehdiloozad et al. [15] propose an LP model for identifying the max projection of units (as reference unit) and demonstrate that the congestion status of $D M U \mathrm{~s}$ is similar to the status congestion of their reference unit. Also, Shadab et al. [16] suggest a novel algorithm through making the connection between the S-shape form of production function and the geometric properties of the anchor points to identify weakly and strongly congested $D M U$ s and congestion amount without determining the efficiency value. Adimi et al. [17] introduce the concept of congestion hyperplane without considering the efficiency value of $D M U$ s to determine congested units. Kerstens and Van de Woestyne [18] illustrate how measuring congestion based on traditional radial input-oriented efficiency measures deals with some limitations and may underestimate the amounts of congestion. Therefore, they use an asymmetric efficiency measure to detect the presence of congestion and measure its amount. Also, they show that the technical efficiency score increases when the production technology exchanges from VRS to CRS assumption, while there is no such relationship when comparing the existence of congestion under the VRS and CRS assumption. In some previous studies, conventional DEA models were proposed to determine congested efficient and inefficient $D M U$ s. Congestion of inefficient $D M U \mathrm{~s}$ is defined at its efficient projection, which is placed on the efficiency frontier. In this case, the output of inefficient units can be increased by keeping their inputs constant and the performance of inefficient units can be improved. Therefore, it is not necessary to explore congested inefficient units.

The features of some models which are compared with the proposed model in this study are summarised in Table 1. In this study, a novel method is proposed to determine congestion of vertex units of production technology in the absence of input disposability postulates. For this purpose, we suggest a new LP model to obtain vertex DMUs. Then, we remove the vertex $D M U$ under evaluation from the production technology. Afterwards, the omitted $D M U$ is compared with other $D M U$ s of the production technology and the status of congestion determined. Besides, we offer a new definition for identifying the congestion status of vertex units and based on it, a novel linear model is proposed that determines congested units with fewer and easier calculations. Lastly, by presenting a theorem, we establish a relationship between the supporting hyperplane of the Production Possibility Set (PPS) and the proposed congestion definition.

In this study, a novel method is proposed to determine congestion of vertex units of production technology in the absence of input disposability postulates. For this purpose, we suggest a new LP model to obtain vertex $D M U$ s. Then, we remove the vertex $D M U$ under evaluation from the production technology. Afterwards, the omitted $D M U$ is compared with other $D M U$ s of the production technology and the status of congestion determined.

We define a new definition for identifying the congestion status of vertex units and, based on it, a novel linear model is proposed that determines congested units with fewer 
and easier calculations. Lastly, by presenting a theorem, we establish a relationship between the supporting hyperplane of the Production Possibility Set (PPS) and the proposed congestion definition.

Table 1. Literature review of some congestion methods

\begin{tabular}{|c|c|c|}
\hline Model features & Structure & Reference \\
\hline $\begin{array}{l}\text { VRS: radial, output-oriented, input disposability, } \\
\text { determining only existence or non-existence of congestion }\end{array}$ & \multirow{3}{*}{$\begin{array}{l}\text { traditional } \\
\text { DEA models }\end{array}$} & {$[6]$} \\
\hline $\begin{array}{l}\text { VRS: output-oriented, non-radial, input disposability, } \\
\text { determining the amount of congestion, } \\
\text { project inefficient } D M U \text { s on BCC-efficiency frontier }\end{array}$ & & [8] \\
\hline $\begin{array}{l}\text { VRS: output-oriented, non-radial, input disposability, } \\
\text { determining the amount of congestion, } \\
\text { project inefficient } D M U \text { s on BCC-efficiency frontier }\end{array}$ & & {$[3]$} \\
\hline $\begin{array}{l}\text { VRS: output-oriented, non-radial, input disposability, } \\
\text { determining the amount of congestion and congestion status, } \\
\text { relationship between congestion and return to scale (RTS), } \\
\text { project inefficient } D M U \text { s on BCC-efficiency frontier }\end{array}$ & \multirow{3}{*}{$\begin{array}{l}\text { two-stage } \\
\text { algorithm }\end{array}$} & {$[12]$} \\
\hline $\begin{array}{l}\text { VRS: output-oriented, non-radial, omitting input disposability, } \\
\text { determining the amount of congestion and congestion status, } \\
\text { project inefficient } D M U \text { s on Tconvex-efficiency frontier }\end{array}$ & & {$[13]$} \\
\hline $\begin{array}{l}\text { VRS: output-oriented, non-radial, omitting input disposability, } \\
\text { determining the amount of congestion and congestion status, } \\
\text { project inefficient } D M U \text { s on Tconvex-efficiency frontier }\end{array}$ & & {$[14]$} \\
\hline $\begin{array}{l}\text { VRS: output-oriented, non-radial, omitting input disposability, } \\
\text { determining the amount of congestion and congestion status, } \\
\text { defining a dominant cone, } \\
\text { determining the max projection of inefficient } D M U \mathrm{~s}\end{array}$ & $\begin{array}{l}\text { traditional } \\
\text { DEA models }\end{array}$ & {$[15]$} \\
\hline
\end{tabular}

The rest of this paper is organised as follows: Section 2 contains some preliminaries. In Section 3, the new model is proposed to detect congestion of a DMU. In Section 4, the results of the proposed models in the case study are provided. Eventually, Section 5 includes some conclusions.

\section{Preliminaries}

The production possibility set (PPS), also called production technology, is defined as the set of all inputs and outputs of a system in which outputs can be produced from the inputs. PPS plays a pivotal role in performance evaluation and productivity analysis. There are several methodologies in the literature to estimate the PPS. Two of the most important methods for estimating the PPS, which are empirically constructed from the 
observations by assuming several postulates, are data envelopment analysis (DEA), and free disposal hull (FDH). DEA is classified as convex technology, while FDH is as nonconvex technology. Throughout the paper, we assume that the dataset fits the convex set.

\subsection{DEA}

Suppose that there is a set of peer observed $D M U \mathrm{~s}\left(D M U_{j}, j=1, \ldots, n\right)$ which each $D M U_{j}$ consumes non-zero input vector $\mathbf{x}_{j}=\left(x_{1 j}, \ldots, x_{m j}\right)$ to produce non-zero output vector $\mathbf{y}_{j}=\left(y_{1 j}, \ldots, y_{s j}\right)$. We also assume that there is not any duplicated $D M U$. Moreover, suppose that each $D M U_{j}, j=1, \ldots, n$ can be expressed in terms of its input and output vectors as $D_{j}=\left(\mathbf{x}_{j}, \mathbf{y}_{j}\right)^{t}$. The superscript $t$ stands for transpose.

The PPS is presented as follows:

$$
\text { PPS }=\{(\mathbf{x}, \mathbf{y}): \mathbf{y} \text { can be produced by } \mathbf{x}\}
$$

Banker et al. [2] introduce the following PPS which is denoted by $T_{v}$, regarding the variable return to scale (VRS) assumption of production technology:

$$
T_{v}=\left\{(\mathbf{x}, \mathbf{y}) \mid \sum_{j=1}^{n} \lambda_{j} x_{j} \leq \mathbf{x}, \quad \sum_{j=1}^{n} \lambda_{j} y_{j} \geq \mathbf{y}, \quad \sum_{j=1}^{n} \lambda_{j}=1, \lambda_{j} \geq 0, \quad j=1, \ldots, n\right\}
$$

$T_{v}$ satisfies the postulates of inclusion of observations, convexity, strong disposability of inputs, strong disposability of outputs and minimum extrapolation. $T_{v}$ is an unbounded convex polyhedral set.

PPS with the elimination of $D M U_{o}$ from the technology of $T_{v}$ can be obtained as follows:

$$
T_{v \backslash o}=\left\{(\mathbf{x}, \mathbf{y}) \mid \sum_{\substack{j=1 \\ j \neq o}}^{n} \lambda_{j} x_{j} \leq \mathbf{x}, \sum_{\substack{j=1 \\ j \neq o}}^{n} \lambda_{j} y_{j} \geq \mathbf{y}, \sum_{\substack{j=1 \\ j \neq o}}^{n} \lambda_{j}=1, \lambda_{j} \geq 0, j=1, \ldots, n, j \neq o\right\}
$$

Let $D M U_{o}$ be the observed activity under evaluation. Banker et al. [2] proposes the following output-oriented model (BCC model) to evaluate the efficiency of $D M U_{o}$ under VRS assumption: 


$$
\begin{aligned}
& \max \varphi+\varepsilon\left(\sum_{i=1}^{m} s_{i}^{-}+\sum_{r=1}^{s} s_{r}^{-}\right) \\
& \text {s.t. } \\
& \sum_{j=1}^{n} \lambda_{j} x_{i j}+s_{i}^{-}=x_{i o}, \quad \forall i \\
& \sum_{j=1}^{n} \lambda_{j} y_{r j}-s_{r}^{+}=\varphi y_{r o}, \quad \forall r \\
& \sum_{j=1}^{n} \lambda_{j}=1 \\
& \lambda_{j}, s_{i}^{-}, s_{r}^{+} \geq 0, \quad \forall j, i, r
\end{aligned}
$$

where $\left(\lambda_{1}, \ldots, \lambda_{n}\right)$ are the intensity variables.

Definition 2.1 (strong efficiency). $D M U_{o}$ is called BCC strong efficient if and only if $\varphi^{*}=1$ and all optimal slacks variables in each alternative optimal solutions is zero, i.e., in all optimal solutions $\mathbf{s}^{+*}=\mathbf{0}$, and $\mathbf{s}^{-*}=\mathbf{0}$. Superscript asterisk $\left(^{*}\right)$ indicates optimality.

Considering $\left(\mathbf{v}, \mathbf{u}, \mathbf{u}_{o}\right)$ as the vector of dual variables associated with constraints of (1), the dual of model (1) can be written as:

$$
\min \sum_{i=1}^{m} v_{i} x_{i o}-u_{o}
$$

s.t.

$$
\begin{aligned}
& \sum_{r=1}^{s} u_{r} y_{r o}=1 \\
& \sum_{r=1}^{s} u_{r} y_{r j}-\sum_{i=1}^{m} v_{i} x_{i j}+u_{o} \leq 0, \quad \forall j \\
& u_{r}, v_{i} \geq \varepsilon, \quad \forall r, i \\
& u_{o} \quad \text { free }
\end{aligned}
$$

The supporting hyperplanes of $T_{v}$ are determined as

$$
H_{\left(\mathbf{u}, \mathbf{v}, \mathbf{u}_{o}\right)}=\left\{(\mathbf{x}, \mathbf{y}) \in R^{m+s} \mid \mathbf{u y}-\mathbf{v} \mathbf{x}+\mathbf{u}_{o}=0\right\}
$$


where $(\mathbf{u}, \mathbf{v}) \neq \mathbf{0}$ is the normal vector and $-\mathbf{u}_{o}$ is the level value of the hyperplane that can be achieved from the model (2). The hyperplane $H_{\left(\mathbf{u}, \mathbf{v}, \mathbf{u}_{o}\right)}$ supports $T_{v}$ at $\left(\mathbf{x}_{o}, \mathbf{y}_{o}\right)$ if $(\mathbf{x}, \mathbf{y}) \in T_{v}$ for each $\mathbf{u y}-\mathbf{v x}+\mathbf{u}_{o} \leq 0$ and $\mathbf{u y}_{o}-\mathbf{v} \mathbf{x}_{o}+\mathbf{u}_{o}=0$.

Definition 2.2 [19]. According to VRS technology, a strong efficient $D M U$ is called a vertex unit, if it cannot be written as any strict convex combination of two distinct points of $T_{v}$.

\subsection{Congestion}

A unit is confronted with congestion if an increase in one or some inputs decreases one or some outputs without worsening the rest of inputs or outputs. The existence of congestion reduces the efficiency of $D M U$ s, so determining it is highly regarded.

According to the input disposability postulate, we have:

$$
(\mathbf{x}, \mathbf{y}) \in T_{v}, \quad \overline{\mathbf{x}} \geq \mathbf{x} \Leftrightarrow(\overline{\mathbf{x}}, \mathbf{y}) \in T_{v}
$$

In the absence of input disposability postulates, Tone and Sahoo [13] introduce a new production technology with VRS assumption as following and named it $T_{\text {convex. }}$.

$$
T_{\text {convex }}=\left\{(\mathbf{x}, \mathbf{y}) \mid \sum_{j=1}^{n} \lambda_{j} x_{j}=\mathbf{x}, \quad \sum_{j=1}^{n} \lambda_{j} y_{j} \geq \mathbf{y}, \quad \sum_{j=1}^{n} \lambda_{j}=1, \lambda_{j} \geq 0, \quad j=1, \ldots, n\right\}
$$

$T_{\text {convex }}$ is a bounded convex set that satisfies the postulates of inclusion of observations, convexity, strong disposability of outputs, and minimum extrapolation.

Definition 2.3 (efficiency). $D M U_{o}$ is called efficient concerning $T_{\text {convex }}$ if and only if in model (3), $\varphi^{*}=1$ and all optimal slacks are zero $\left(\mathbf{s}^{+^{*}}=\mathbf{0}\right)$ [13].

$$
\begin{aligned}
& \max \varphi+\varepsilon \sum_{r=1}^{s} s_{r}^{+} \\
& \text {s.t. } \\
& \sum_{j=1}^{n} \lambda_{j} x_{i j}=x_{i o}, \quad \forall i \\
& \sum_{j=1}^{n} \lambda_{j} y_{r j}=\varphi y_{r o}+s_{r}^{+}, \quad \forall r \\
& \sum_{j=1}^{n} \lambda_{j}=1 \\
& \lambda_{j}, s_{r}^{+} \geq 0, \quad \forall j, r
\end{aligned}
$$


PPS with the elimination of $D M U_{o}$ from the technology of $T_{\text {convex }}$ can be obtained as below:

$$
T_{\text {convex } \backslash o}=\left\{(\mathbf{x}, \mathbf{y}) \mid \sum_{\substack{j=1 \\ j \neq o}}^{n} \lambda_{j} x_{j}=\mathbf{x}, \sum_{\substack{j=1 \\ j \neq o}}^{n} \lambda_{j} y_{j} \leq \mathbf{y}, \sum_{\substack{j=1 \\ j \neq o}}^{n} \lambda_{j}=1, \lambda_{j} \geq 0, j=1, \ldots, n, j \neq o\right\}
$$

Many methods have been developed to investigate congestion by solving the conventional DEA models. One of these studies is by Tone and Sahoo [13]. They propose an algorithm to find out congested $D M U$ s and identify the status of congestion via proposing the concept of 'strong congestion' and 'weak congestion' as follows for the first time.

Definition 2.4 (strong congestion). $D M U_{o}$ is strongly congested if it is an efficient unit with respect to $T_{\text {convex }}$ and there exists an activity $(\overline{\mathbf{x}}, \overline{\mathbf{y}}) \in T_{\text {convex }}$ such that $\overline{\mathbf{x}}=\alpha \mathbf{x}_{o}(0<\alpha<1)$ and $\overline{\mathbf{y}} \geq \beta \mathbf{y}_{o}(\beta>1)$.

Definition 2.5 (weak congestion). $D M U_{o}$ is weakly congested if there exists an activity $(\overline{\mathbf{x}}, \overline{\mathbf{y}}) \in T_{\text {convex }}$ that consumes fewer resources in one or more inputs to produce more products in some outputs, i.e., $\hat{\mathbf{x}} \leq \mathbf{x}_{o}, \hat{\mathbf{y}} \geq \mathbf{y}_{o}$ and $\hat{\mathbf{y}} \neq \mathbf{y}_{o}$.

\section{Identifying congestion in DEA}

In this section, we propose a new method to identify congested $D M U$ s and determine the status of congestion (strong or weak). To this aim, a vertex unit of $T_{\text {convex }}$ is eliminated then if there exists an activity in $T_{\text {convex }}$ with lower inputs and more outputs as compared with omitted unit, we say vertex unit evidences congestion.

Regarding to $T_{\text {convex }}$ a vertex unit can be defined as below.

Definition 3.1 (vertex unit). An efficient $D M U_{o}=\left(\mathbf{x}_{o}, \mathbf{y}_{o}\right)$ is a vertex unit of $T_{\text {convex }}$ if it is an extreme point, and the set of vertex points of $T_{\text {convex }}$ is denoted by $E T_{\text {convex }}$.

According to the following theorem, vertex units of $T_{\text {convex }}$ can be obtained. Notice that the objective function of model (4) is to minimise the intensity variable associated with $D M U_{o}$ (the unit under assessment). 
Theorem 3.1. $D M U_{o}=\left(\mathbf{x}_{o}, \mathbf{y}_{o}\right)$ is a vertex unit of $T_{\text {convex }}$ if and only if the optimal objective function of model (4) is equal to one $\left(\lambda_{o}^{*}=1\right)$, and it is not an extreme efficient unit if and only if $\lambda_{o}^{*}=0$.

$$
\begin{aligned}
& \lambda_{o}^{*}=\min \lambda_{o} \\
& \text { s.t. } \\
& \sum_{j=1}^{n} \lambda_{j} x_{i j}=x_{i o}, \quad \forall i \\
& \sum_{j=1}^{n} \lambda_{j} y_{r j} \geq y_{r o}, \quad \forall r \\
& \sum_{j=1}^{n} \lambda_{j}=1 \\
& \lambda_{j} \geq 0, \quad \forall j
\end{aligned}
$$

Proof. Suppose that $D M U_{o}=\left(\mathbf{x}_{o}, \mathbf{y}_{o}\right)$ is a vertex unit of $T_{\text {convex. }}$ Let $\lambda^{*}=\left(\lambda_{1}^{*}, \ldots, \lambda_{n}^{*}\right)$ be an optimal solution of model (4). On the contrary, assume that $0<\lambda_{o}^{*}<1$ or $\lambda_{o}^{*}=0$. It follows from (4.3) that $\lambda_{o}^{*}<1$. According to constraint (4.1), we have: $\sum_{j=1}^{n} \lambda_{j}^{*} x_{i j}=x_{i o}$. It can be written as $\sum_{\substack{j=1 \\ j \neq o}}^{n} \lambda_{j}^{*} x_{i j}+\lambda_{o}^{*} x_{i o}=x_{i o}$. Therefore,

$$
\sum_{\substack{j=1 \\ j \neq 0}}^{n} \lambda_{j}^{*} x_{i j}=x_{i o}-\lambda_{o}^{*} x_{i o}=\left(1-\lambda_{o}^{*}\right) x_{i o}
$$

Since $1-\lambda_{o}^{*}>0$, then dividing by $1-\lambda_{o}^{*}$, we have $\sum_{\substack{j=1 \\ j \neq 0}}^{n} \frac{1-\lambda_{j}^{*}}{\left(1-\lambda_{o}^{*}\right)} x_{i j}=x_{i o}$.

Moreover, according to constraint (4.2), we have $\sum_{j=1}^{n} \lambda_{j}^{*} y_{r j} \geq y_{r o}$. Equivalently, it can be written as $\sum_{\substack{j=1 \\ j \neq o}}^{n} \lambda_{j}^{*} y_{r j}+\lambda_{o}^{*} y_{r o} \geq y_{r o}$. So, $\sum_{\substack{j=1 \\ j \neq o}}^{n} \lambda_{j}^{*} y_{r j} \geq\left(1-\lambda_{o}^{*}\right) y_{r o}$. 
Obviously, so dividing by $1-\lambda_{o}^{*}$ we have: $\sum_{\substack{j=1 \\ j \neq o}}^{n} \frac{\lambda_{j}^{*}}{1-\lambda_{o}^{*}} y_{r j} \geq y_{r o}$. Also, from constrain (4.3), we have $\sum_{j=1}^{n} \lambda_{j}^{*}=1$, then $\sum_{\substack{j=1 \\ j \neq o}}^{n} \lambda_{j}^{*}+\lambda_{o}^{*}=1$. Therefore, $\sum_{\substack{j=1 \\ j \neq o}}^{n} \frac{\lambda_{j}^{*}}{1-\lambda_{o j}^{*}}=1$.

Therefore, model (4) has a solution as $\lambda_{j}=\lambda_{j}^{*} /\left(1-\lambda_{o}^{*}\right), \lambda_{o}=0$ for $j=1, \ldots, n, j \neq o$. Note that if $\lambda_{o}^{*}=0$ then

$$
\begin{aligned}
& \sum_{\substack{j=1 \\
j \neq o}}^{n} \lambda_{j}^{*} x_{i j}=x_{i o} \\
& \sum_{\substack{j=1 \\
j \neq o}}^{n} \lambda_{j}^{*} y_{r j} \geq y_{r o}
\end{aligned}
$$

We consider two subcases from the constrain (6):

\section{Case 1}

$$
\sum_{\substack{j=1 \\ j \neq o}}^{n} \lambda_{j}^{*} y_{r j}=y_{r o}
$$

Considering constrains (5) and (7) contradict extreme point of $D M U_{o}$ since it can be written as any strict convex combination of the rest distinct points of $T_{\text {convex }}$.

\section{Case 2}

$$
\sum_{\substack{j=1 \\ j \neq o}}^{n} \lambda_{j}^{*} y_{r j}>y_{r o}
$$

If Case 1 happens, then $D M U_{o}$ is not a vertex unit (note that we do not have any duplicated $D M U$ ). If Case 2 happens, then it is not difficult to show that $D M U_{o}$ is not efficient. These contradict the assumptions. Therefore, $\lambda_{o}^{*}=1$.

Assume that $\lambda_{o}^{*}=1$. On the contrary, suppose that $D M U_{o}$ is not a vertex unit of $T_{\text {convex. }}$ Thus, it can be written as a strict convex combination of the rest of DMUs. So, there exists $\lambda=\left(\lambda_{1}, \ldots, \lambda_{o-1}, \lambda_{o+1}, \ldots, \lambda_{n}\right)$ such that $\sum_{\substack{j=1 \\ j \neq o}}^{n} \lambda_{j} x_{i j}=x_{i o}, \sum_{\substack{j=1 \\ j \neq o}}^{n} \lambda_{j} y_{r j} \geq y_{r o}, \sum_{\substack{j=1 \\ j \neq o}}^{n} \lambda_{j}=1$. Thus, $\lambda_{o}=0$ 
and $\lambda=\left(\lambda_{1}, \ldots, \lambda_{o-1}, \lambda_{o+1}, \ldots, \lambda_{n}\right)$ is a feasible solution to model (4). The objective value of (4) associated with this feasible solution is zero. This contradicts the optimality of $\lambda_{o}^{*}=1$ and the proof is complete.

To find out congested $D M U$, we propose the following definition:

Definition 3.2 (congestion in DEA). Let $D M U_{o}=\left(\mathbf{x}_{o}, \mathbf{y}_{o}\right)$ be a vertex point of $T_{\text {convex }}$. The next two statements define the weak and strong congestion, respectively.

I. $D M U_{o}=\left(\mathbf{x}_{o}, \mathbf{y}_{o}\right)$ evidences weak congestion if there exists some $(\hat{x}, \hat{y}) \in T_{\text {convexlo }}$ such that $\hat{\mathbf{x}} \leq \mathbf{x}_{o}$ and $\hat{\mathbf{y}} \geq \mathbf{y}_{o}, \hat{\mathbf{y}} \neq \mathbf{y}_{o}$.

II. $D M U_{o}=\left(\mathbf{x}_{o}, \mathbf{y}_{o}\right)$ evidences strong congestion if there exists some $(\hat{x}, \hat{y}) \in T_{\text {convex } \ o}$ such that $\hat{\mathbf{x}}<\mathbf{x}_{o}$ and $\hat{\mathbf{y}}>\mathbf{y}_{o}$.

By the following definition, the applied method to determine congested units is introduced:

Definition 3.3. Let $D M U_{o}=\left(\mathbf{x}_{o}, \mathbf{y}_{o}\right)$ be a vertex unit of $T_{\text {convex }}$. It is facing strong (weak) congestion if and only if in model (9) $\varphi^{*}>1$ and $s_{i}^{-*}>0\left(s_{i}^{-*} \geq 0\right.$ and $\left.s_{i}^{-*} \neq 0\right)$ )for $i \in\{1, \ldots, m\}$ in any alternative optimal solution of (9).

$$
\begin{aligned}
& \varphi^{*}=\max \varphi+\varepsilon \sum_{i=1}^{m} s_{i} \\
& \text { s.t. } \\
& \sum_{\substack{j=1 \\
j \neq o}}^{n} \lambda_{j} x_{i j}=x_{i o}-s_{i}^{-}, \quad \forall i \\
& \sum_{\substack{j=1 \\
j \neq o}}^{n} \lambda_{j} y_{r j} \geq \varphi y_{r o} \\
& \sum_{\substack{j=1 \\
j \neq o}}^{n} \lambda_{j}=1 \\
& \lambda_{j}, s_{i} \geq 0, \quad \forall i, j, j \neq o
\end{aligned}
$$

Through the following theorem, we show that the definitions (3.2) and (3.3) are equivalent.

Theorem 3.2. Let $D M U_{o}=\left(\mathbf{x}_{o}, \mathbf{y}_{o}\right) \in E T_{\text {convex }}$. $D M U_{o}$ is strongly (weakly) congested according to definition 3.2 if and only if the optimal value of an objective function of model (9) is positive, i.e., $\varphi^{*}>1$ and $s_{i}^{-*}>0 \quad\left(s_{i}^{-*} \geq 0\right.$ and $\left.s_{i}^{-*} \neq 0\right)$ for $i \in\{1, \ldots, m\}$. 
Proof. Let $D M U_{o}=\left(\mathbf{x}_{o}, \mathbf{y}_{o}\right) \in E T_{\text {convex }}$ and $\varphi^{*}>1$ and $s_{i}^{-*}>0$ for $i \in\{1, \ldots, m\}$. Assume that $\left(\varphi^{*}, \lambda^{*}, \mathbf{s}^{-*}\right)$ is optimal solution of model (9). Besides, we consider all inputs and outputs are positive, i.e., $\left(\mathbf{x}_{o}, \mathbf{y}_{o}\right)>\mathbf{0}$ therefore:

$$
\begin{gathered}
\hat{\mathbf{y}}=\sum_{\substack{j=1 \\
j \neq o}}^{n} \lambda_{j}^{*} y_{j} \geq \varphi^{*} \mathbf{y}_{o}>\mathbf{y}_{o} \\
\hat{\mathbf{x}}=\sum_{\substack{j=1 \\
j \neq o}}^{n} \lambda_{j}^{*} x_{j}=\mathbf{x}_{o}-s^{-*}<\mathbf{x}_{o}
\end{gathered}
$$

Constraint (11) implies that $\hat{\mathbf{x}}=\sum_{\substack{j=1 \\ j \neq o}}^{n} \lambda^{*}{ }_{j} x_{k j}=x_{k o}-s_{k}{ }^{-*}<x_{k o}$ for some $k \in\{1, \ldots, m\}$. Due to constraints (10) and (11), we have an activity ( $\hat{\mathbf{x}}, \hat{\mathbf{y}})$ such that $\hat{\mathbf{y}}>\mathbf{y}_{o}$ and $\hat{\mathbf{x}}<\mathbf{x}_{o}$. Therefore, as based on definition (3.2), $D M U_{o}$ has strong congestion.

Assume that $D M U_{o}$ is strongly congested. Let $\left(\varphi^{*}, \lambda^{*}, \mathbf{s}^{-*}\right)$ be an optimal solution to model (9). According to definition (3.2), there exist an activity $(\hat{\mathbf{x}}, \hat{\mathbf{y}})$ such that $\hat{\mathbf{y}}>\mathbf{y}_{o}$ and $\hat{\mathbf{x}}<\mathbf{x}_{o}$. As $(\hat{\mathbf{x}}, \hat{\mathbf{y}}) \in T_{\text {convex } \backslash o}$, there exists some $\hat{\lambda}=\left(\hat{\lambda}_{1}, \ldots, \hat{\lambda}_{n}\right) \geq \mathbf{0}$ such that:

$$
\begin{gathered}
\sum_{\substack{j=1 \\
j \neq o}}^{n} \hat{\lambda}_{j} y_{j} \geq \hat{\mathbf{y}}>\mathbf{y}_{o} \\
\hat{\mathbf{x}}=\sum_{\substack{j=1 \\
j \neq o}}^{n} \hat{\lambda}_{j} x_{j}<\mathbf{x}_{o}
\end{gathered}
$$

Inequality (13) converts to $\sum_{\substack{j=1 \\ j \neq 0}}^{n} \hat{\lambda}_{j} x_{i j}+\hat{s}_{i}^{-}=x_{i o}$ with $\hat{s}_{i}^{-}>0$ for each $i \in\{1, \ldots, m\}$.

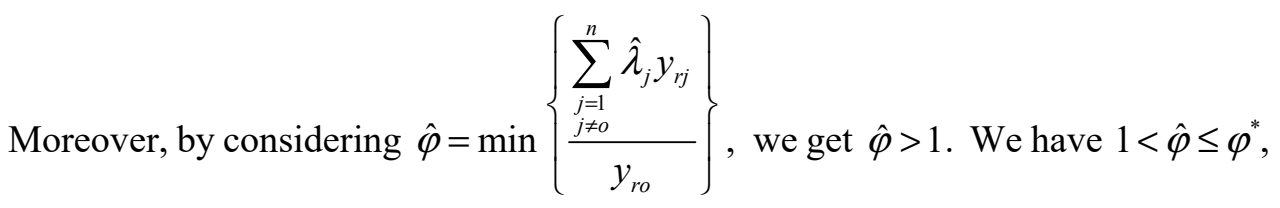
since the objective function of model (9) is in the maximising form.

This completes the proof. The theorem can be proved for weak congestion easily. Figure1 depicts the proposed model graphically. 


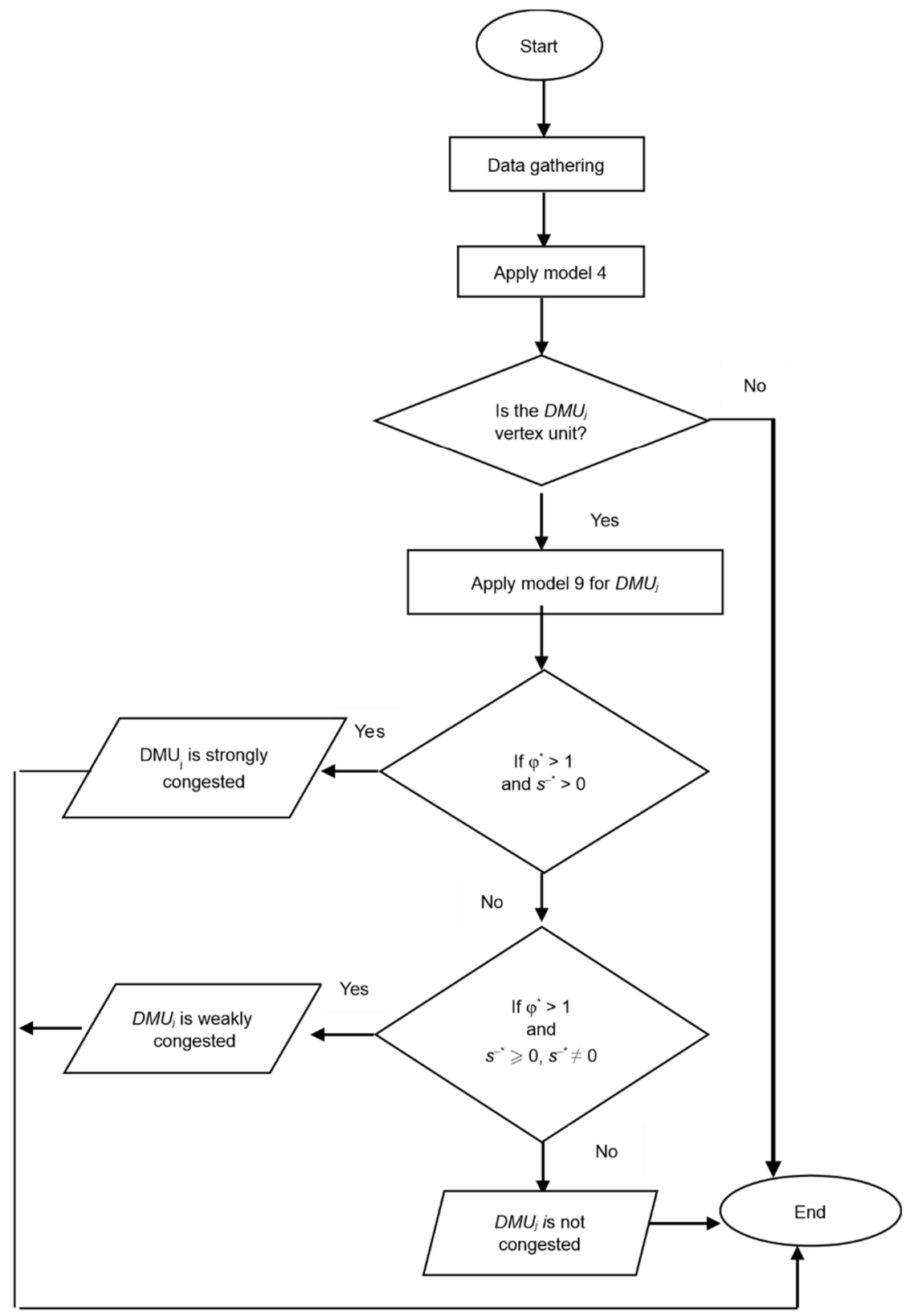

Fig. 1. The process of determining congested DMUs

Example 3.1. Now, we use Theorem 3.1 with the help of a numerical example originally used by Cooper et al. [9]. Consider $8 D M U$ s with single input $(I)$ and single output $(O)$ 
whose data set. Model (3) is solved to determine vertex units with respect to $T_{\text {convex }}$. Results are shown in Table 2. As can be seen, $D M U_{A}, D M U_{B}, D M U_{C}$, and $D M U_{D}$ are vertex units $\left(\lambda_{j}^{*}=1, j=A, B, C, D\right)$ and others are not. Now, we apply the proposed model for these vertex $D M U$ s. Model (4) is run and optimal solution is also shown in Table 2. According to Theorem (3.2), only $D M U_{D}$ has congestion because of $\varphi_{D}{ }^{*}>1$ and $s^{-*}>0$.

Table 2. Dataset of $8 D M U$ s and results of models (3) and (4)

\begin{tabular}{|c|c|c|c|c|c|c|c|}
\hline$D M U$ & $I$ & $O$ & $\lambda^{*}$ & $\varphi^{*}$ & $s^{-*}$ & $\begin{array}{c}\text { Vertex } \\
\text { units }\end{array}$ & $\begin{array}{c}\text { Congested } \\
\text { units }\end{array}$ \\
\hline$A$ & 1 & 0.5 & 1 & infeasible & - & $\checkmark$ & $\times$ \\
\hline$B$ & 2 & 2 & 1 & 0.62 & 0 & $\checkmark$ & $\times$ \\
\hline$C$ & 3 & 2 & 1 & 1 & 1 & $\checkmark$ & $\times$ \\
\hline$D$ & 5 & 1 & 1 & 2 & 2 & $\checkmark$ & $\checkmark$ \\
\hline$E$ & 4 & 1 & 0 & - & - & $\times$ & - \\
\hline$F$ & 4 & 1.2 & 0 & - & - & $\times$ & - \\
\hline$G$ & 4.5 & 1.2 & 0 & - & - & $\times$ & - \\
\hline$H$ & 3 & 1 & 0 & - & - & $\times$ & - \\
\hline
\end{tabular}

Now, regarding hyperplane properties and congestion definition, we propose the following definition:

Definition 3.4. Let $D M U_{o}=\left(\mathbf{x}_{o}, \mathbf{y}_{o}\right) \in E T_{\text {convex }} . D M U_{o}$ has congestion if there exists a hyperplane $H_{\left(\mathbf{u}, \mathbf{v}, \mathbf{u}_{o}\right)}=\left\{(\mathbf{x}, \mathbf{y}) \in R^{m+s} \mid \mathbf{u y}-\mathbf{v x}+\mathbf{u}_{o}<0\right\}$ that supporting $T_{\text {convex }}$ with a normal vector $(\mathbf{u}, \mathbf{v})$ such that at least one component of vector $v_{i}^{*}$ for $i=\{1, \ldots, m\}$ is zero.

Theorem 3.3. Definitions (3.4) and (3.3) are equivalent.

Proof. Let definition (3.3) hold and $\left(\varphi^{*}, s^{-*}, \lambda^{*}\right)$ be the optimal solution of model (9). Dual of the model (9) is as follows:

$\min v x_{o}+u_{o}$

s.t.

$u y_{o}=1$

$u y_{j}-v x_{j}-u_{o} \leq 0, \forall j, j \neq o$

$u \geq 0, \quad v \geq 0$

$u_{o}: U R S$ 
Suppose that $\left(\mathbf{u}^{*}, \mathbf{v}^{*}, \mathbf{u}_{o}^{*}\right)$ is an optimal solution of model (14). Based on the fundamental theorem of duality, $\varphi^{*}=v^{*} x_{o}+u_{o}^{*}$

Since $\varphi^{*}>1$ so $v^{*} x_{o}+u_{o}^{*}=\varphi^{*}>1=u^{*} y_{o} \rightarrow u^{*} y_{o}-v^{*} x_{o}+u_{o}^{*}<0$. Therefore, $D M U_{o}$ $=\left(\mathbf{x}_{o}, \mathbf{y}_{o}\right)$ belongs to hyperplane $H$. According to the complementary slackness theorem, we have $v_{i}^{*} s_{i}^{-*}=0$ for each, at least one $i \in\{1, \ldots, m\}$ for some $s_{i}^{-*} \neq 0$, and $s_{i}^{-*} \geq 0$. Since $i \in\{1, \ldots, m\}$, component of $v^{*}$ equals zero, i.e., $v^{*}=0$ for some $i \in\{1, \ldots, m\}$.

Let $D M U_{o}=\left(\mathbf{x}_{o}, \mathbf{y}_{o}\right)$ be a vertex point of $T_{\text {convex }}$, and there exists a hyperplane $H_{\left(\mathbf{u}, \mathbf{v}, \mathbf{u}_{o}\right)}$ supporting $T_{\text {convex }}$ such that at least one component of vector $v$ is zero. So, $u^{*} y_{o}-v^{*} x_{o}+u_{o}^{*}<0$.

Defining $u^{*} y_{o}=\alpha$ and $\frac{u^{*}}{\alpha} y_{o}=1$, therefore $\frac{u^{*}}{\alpha} y_{o}-\frac{v^{*}}{\alpha} x_{o}+\frac{u_{o}^{*}}{\alpha}<0$, then $\frac{u^{*}}{\alpha} y_{o}$ $<\frac{v^{*}}{\alpha} x_{o}-\frac{u_{o}^{*}}{\alpha}$. Therefore, $1=\frac{u^{*}}{\alpha} y_{o}<\frac{v^{*}}{\alpha} x_{o}-\frac{u_{o}^{*}}{\alpha} \leq \varphi^{*}$.

Hence, $\frac{u^{*}}{\alpha}, \frac{v^{*}}{\alpha}, \frac{u_{o}{ }^{*}}{\alpha}$ is a feasible solution to model (14). Based on the status of objective function, we have $\varphi^{*}>\varphi>1$, and based on complementary slackness theorem, $s_{i}^{-*}+\frac{v_{i}^{*}}{\alpha}>0$. Since $v_{i}^{*}=0$, then $s_{i}^{-*}>0$ for some $i \in\{1, \ldots, m\}$. These complete the proof.

\section{Empirical example}

In this section, we evaluate congested inputs of $D M U$ s and compare the results of the proposed model with the results of some previous models. For this purpose, we consider a set of chain stores in Japan for a period of 27 years from 1975 to 2001 [13]. The years under evaluation are considered as $D M U$ s. Chain stores consume two inputs to produce one output. Two inputs $\left(I_{1}\right.$ and $\left.I_{2}\right)$ are the numbers of stores and total area of stores (unit: $1000 \mathrm{~m}^{2}$ ). The output $(O)$ is annual sales (unit: hundred million yen). The value of two inputs and one output of chain stores in 1975 to 2001 are shown in Table 3. According to the data, the number of chain stores increases steadily until 1993, after which the trend consistently decreases. Except for the last year, the area of stores is consistently rising throughout. This increase takes place in the first half of years with a smaller ratio and in the second half with a higher ratio. This shows that with the disappearance of small stores, the rapid growth of large stores in recent years is significant. The annual sales trend increases until 1996 and consistently declines in remaining years. Now, our presented model is implemented in this example to determine the years when chain 
stores evidence congestion. Then, we compare the results of our proposed model with some of the methods presented in earlier studies. According to our congestion definition, congested $D M U$ s are a subset of vertex $D M U$ s of $T_{\text {convex }}$ which can be achieved through model (4). Therefore, model (4) is run and the results are reported in Table 4. As can be seen, the years 1975, 1976, 1988, 1991-1993, 1996, 1998, and 2000 are determined as vertex DMUs $\left(\lambda^{*}=1\right)$, and the remaining years identified as inefficient $D M U$ s $\left(\lambda^{*}=0\right)$. Then, model (9) is solved to find out congested vertex $D M U$ s. The optimal solution of model (9) is shown in Table 4. The columns $s_{1}^{-*}$ and $s_{2}^{-*}$ indicate excesses in the first and second inputs, respectively. Model (9) is infeasible for the year 1975. In the year $2000\left(D M U_{26}\right)$, chain stores encounter strong congestion because both input slacks are greater than zero and the optimal objective function of model (9) is greater than one $\left(\varphi^{*}=1.02, s_{1}^{*}=2.26, s_{2}^{*}=1.23\right)$. It means that there exists an activity that belongs to this technology and that produces more output $(162847 \times 1.02=166103)$ by consuming less first input $(7053-2.26=7050.74)$ and the second input $(19698$ $-1.23=19696.77$ ) compared to $D M U_{26}$.

It shows that $D M U_{26}$ can obtain more outputs by consuming less of its inputs. It is noticeable that congestion was due to the excess in the numbers of stores and the area of stores. In the years 1976, 1988, 1992, and 1993 the Japanese chain stores evidence weak congestion (only one of the two input slacks $\left(s_{1}^{-*}\right)$ is greater than zero and the objective function of model (9) is greater than one for these years). It reveals that they were able to achieve more sales by reducing the number of stores during these years. By solving model (1) and in terms of definition 2.1, we explore that the chain stores in the years 1991, 1996, and 1998 are BCC-efficient and, hence, not congested. They perform well during these years.

Table 3. Data set of Japanese chain stores [14]

\begin{tabular}{|c|c|c|c|c|c|c|c|}
\hline$D M U$ & $I_{1}$ & $I_{2}$ & $O$ & $D M U$ & $I_{1}$ & $I_{2}$ & $O$ \\
\hline 1975 & 2412 & 5480 & 41091 & 1989 & 6829 & 11717 & 131862 \\
\hline 1976 & 3163 & 6233 & 48367 & 1990 & 6995 & 11987 & 140817 \\
\hline 1977 & 3350 & 6798 & 56000 & 1991 & 7338 & 12463 & 150583 \\
\hline 1978 & 3371 & 7274 & 60940 & 1992 & 7946 & 13426 & 152943 \\
\hline 1979 & 3778 & 7992 & 69046 & 1993 & 8236 & 14147 & 155128 \\
\hline 1980 & 4020 & 8500 & 77347 & 1994 & 7722 & 15014 & 158714 \\
\hline 1981 & 5029 & 9246 & 85805 & 1995 & 7727 & 15022 & 161739 \\
\hline 1982 & 5164 & 9639 & 90433 & 1996 & 7822 & 16191 & 169786 \\
\hline 1983 & 5285 & 9981 & 95640 & 1997 & 7531 & 16969 & 167195 \\
\hline 1984 & 5618 & 10276 & 100257 & 1998 & 7201 & 17627 & 167187 \\
\hline 1985 & 5981 & 10521 & 105944 & 1999 & 7281 & 18364 & 165480 \\
\hline 1986 & 6217 & 10766 & 109857 & 2000 & 7053 & 19698 & 162847 \\
\hline 1987 & 6455 & 11144 & 116114 & 2001 & 6067 & 16176 & 154671 \\
\hline 1988 & 6674 & 11418 & 125404 & & & & \\
\hline
\end{tabular}


Table 4. Results of models (4) and (9)

\begin{tabular}{|c|c|c|c|c|}
\hline$D M U$ & $\lambda^{*}$ & $\varphi^{*}$ & $s_{1}^{-*}$ & $s_{2}^{-*}$ \\
\hline 1975 & \multirow{10}{*}{1} & infeasible & - & - \\
\hline 1976 & & 1.09 & 219.81 & 0 \\
\hline 1988 & & 1.07 & 73.17 & 0 \\
\hline 1991 & & 0.93 & 2.03 & 0 \\
\hline 1992 & & 1.02 & 482.97 & 0 \\
\hline 1993 & & 1.03 & 679.37 & 0 \\
\hline 1996 & & 0.96 & 582.90 & 0 \\
\hline 1998 & & 0.98 & 0 & 0 \\
\hline 2000 & & 1.02 & 2.26 & 1.23 \\
\hline 2001 & & 0.89 & 0 & 0 \\
\hline
\end{tabular}

Lastly, we implement the models proposed by Färe et al. [6], Wei and Yan [12], Tone and Sahoo [13], Sueyoshi and Sekitiani [14], Cooper et al [9], Mehdiloozad et al. [15] for the data from Table 3 and compare the results with the proposed model. Results are presented in Table 5. $\checkmark$ indicates the presence of congestion. FGL is a two-stage approach. In the first step, the BCC-efficiency of $D M U_{o}$ is calculated, and in the second step, by omitting the input disposability postulates from PPS, the efficiency of $D M U_{o}$ is calculated. The model is a radial approach that calculates the congestion impacts as the ratio of the observed amounts to expected amounts. For detecting congested $D M U$ through BCSW approach, the projection point of $D M U_{o}$ in output-oriented is determined. Afterwards, the outputs are fixed to that projection point, and the maximum amounts of inputs that can be augmented to the projection's points are computed. Then, the difference between each pair of input slacks, and the maximum amount is the amount of congestion. One model of Cooper integrates a two-stage model of the BCSW in one model and identifies the congested DMUs. Wei and Yan [12] restrict their study to the efficient $D M U$ s which are on the boundary of $T_{\text {convex }}$, and inefficient $D M U$ s are depicted on the boundary of $T_{\text {convex }}$. Then, they suggest a two-step algorithm based on conventional DEA models on each step of which one LP model should be solved. Also, they show that a $D M U$ evidences congestion, if and only if it is not BCC output efficient. According to Tone and Sahoo's approach, $D M U$ s project on a strongly efficient boundary of $T_{\text {convex }}$, then by proposing a two-stage algorithm, strong or weak congested $D M U$ s are identified. Sueyoshi and Sekitiani propose wide congestion, conceptually covering both strong and weak congestion, and then a two-stage algorithm based DEA models which can uniquely determine the congested DMUs. Mehdiloozad et al. [36] propose an LP model for identifying the Max projection of units (as reference unit) and demonstrate that the congestion status of $D M U$ s is similar to the status congestion of their reference unit. Mehdiloozad et al. [36] determine a dominant cone for $D M U_{o}$ and

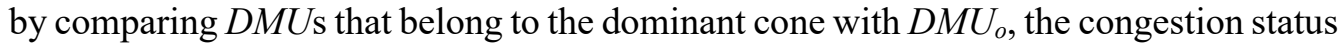
of $D M U_{o}$ can be obtained. 
Lastly, congested units among vertex $D M U$ s that belong to $T_{\text {convex }}$ are shown in the last column of Table 5. By comparing the results, it can be concluded that all congested vertex $D M U$ s of $T_{\text {convex }}$ are the same in all methods. One of the advantages of our proposed method is that by solving only one LP model and less and easier calculation, the congestion status of vertex $D M U$ s can be detected. Of course, no one can claim which method is better because all methods are presented with different definitions.

Table 5. Results of some methods for existence and non-existence of congestion

\begin{tabular}{|c|c|c|c|c|c|c|c|c|}
\hline$D M U$ & FGL & $\begin{array}{l}\text { Wei } \\
\text { and } \\
\text { Yan }\end{array}$ & $\begin{array}{l}\text { Tone } \\
\text { and } \\
\text { Sahoo }\end{array}$ & $\begin{array}{l}\text { Sueyoshi } \\
\text { and } \\
\text { Sekitiani }\end{array}$ & $\begin{array}{l}\text { BCSW } \\
\text { model }\end{array}$ & $\begin{array}{c}\text { One-model } \\
\text { Cooper }\end{array}$ & Mehdiloozad et al. & $\begin{array}{c}\text { Proposed } \\
\text { model }\end{array}$ \\
\hline 1975 & & & & & & & & infeasible \\
\hline 1976 & $\checkmark$ & $\checkmark$ & \multirow{7}{*}{ weak } & $\checkmark$ & $\checkmark$ & $\checkmark$ & \multirow{7}{*}{ weak } & weak \\
\hline 1977 & & $\checkmark$ & & $\checkmark$ & $\checkmark$ & $\checkmark$ & & \\
\hline 1985 & & $\checkmark$ & & $\checkmark$ & $\checkmark$ & $\checkmark$ & & \\
\hline 1986 & $\checkmark$ & $\checkmark$ & & $\checkmark$ & $\checkmark$ & $\checkmark$ & & \\
\hline 1987 & $\checkmark$ & $\checkmark$ & & $\checkmark$ & $\checkmark$ & $\checkmark$ & & \\
\hline 1988 & $\checkmark$ & $\checkmark$ & & $\checkmark$ & $\checkmark$ & $\checkmark$ & & weak \\
\hline 1989 & & $\checkmark$ & & $\checkmark$ & $\checkmark$ & $\checkmark$ & & \\
\hline 1992 & $\checkmark$ & $\checkmark$ & strong & & $\checkmark$ & $\checkmark$ & strong & weak \\
\hline 1993 & $\checkmark$ & $\checkmark$ & \multirow{3}{*}{ weak } & $\checkmark$ & $\checkmark$ & $\checkmark$ & \multirow{3}{*}{ weak } & weak \\
\hline 1994 & & $\checkmark$ & & $\checkmark$ & $\checkmark$ & $\checkmark$ & & \\
\hline 1995 & & $\checkmark$ & & $\checkmark$ & $\checkmark$ & $\checkmark$ & & \\
\hline 1997 & & $\checkmark$ & \multirow{3}{*}{ strong } & $\checkmark$ & $\checkmark$ & $\checkmark$ & \multirow{3}{*}{ strong } & \\
\hline 1999 & $\checkmark$ & $\checkmark$ & & $\checkmark$ & $\checkmark$ & $\checkmark$ & & \\
\hline 2000 & $\checkmark$ & $\checkmark$ & & $\checkmark$ & $\checkmark$ & $\checkmark$ & & strong \\
\hline
\end{tabular}

\section{Conclusion}

The presence of congestion in a $D M U$ reduces its efficiency, so the determination of congestion is of great significance. Some studies were conducted to detect the congestion via solving conventional DEA models. In this study, we define vertex DMUs of $T_{\text {convex }}$ and propose an LP model to obtain vertex units. Then, we propose a conventional DEA model to identify weakly or strongly congested vertex units.

In the suggested model, we remove a vertex unit from the production technology of $T_{\text {convex. }}$ If there is an activity in the production technology whose all input and output components are less and more of inputs and outputs of the omitted unit, we say the deleted unit has strong congestion. Moreover, if there is an activity that consumes fewer resources in one or more inputs to produce more products in some outputs, we claim that the omitted unit evidences weak congestion. In this study, congested $D M U$ s can be obtained easily and by solving only one LP with no need to calculate the efficiency 
value of $D M U$ s. Finally, our model is implemented to identify congested Japanese chain stores from 1975 to 2001. In this example, the years are considered as DMUs. First, we determine vertex $D M U$ s. As based on the results obtained from solving the model (4), ten years are obtained as vertex $D M U$ s. Then, model (9) is run for vertex $D M U$ s. According to Theorem 3.2, chain stores evidence strong congestion in the year 2000 and weak congestion in the years 1976, 1988, 1992, and 1993. In only three years, 1991, 1996 and 1998, chain stores did well, and no congestion occurred.

Results obtained from comparing previous models show that congested vertex $D M U$ s obtained from our model are similar to the models proposed by Wei and Yan method [12], Tone and Sahoo method [13], Sueyoshi and Sekitiani method [14], BCSW method [8], Mehdiloozad et al. method [15] and Cooper model [9]. Since each model has a specific definition of congestion, it cannot be said which model is more preferable to the others. Generally, the chain stores are extremely efficient only in the third evaluating period. Also, during the vertex years, chain stores did not evidence congestion for solely three years. In 2000 , the chain stores faced strong congestion that made them reduce both inputs as much as slack variables 2.26 and 1.23 , respectively, to improve their performance. In the years 1976, 1988, 1992, and 1993 the chain stores evidenced weak congestion in the first input that should reduce their first input as much as slack variable $219.81,73.17,482.97,679.37,582.90$ and 2.26 , respectively, to extend their efficiency.

In this study, the suggested model allows managers to monitor the state of their companies along with discovering congested units.

The contributions of this study are as follows:

- detecting the vertex units, a novel LP model is proposed;

- exploring the congested vertex units and congestion status; only one new LP model is suggested and congested DMUs are identified with easier and fewer calculations.

Further research can be done based on the results of this paper. Some of these can be as follows:

- the same model can be developed for finding congested $D M U$ s with network structure;

- developing the proposed model to identify congested $D M U$ s in the presence of fuzzy or interval data.

\section{References}

[1] Charnes A., CoOper W.W., Rhodes E., Measuring the efficiency of decision-making units, Eur. J. Oper. Res., 1978, 2, 429-444.

[2] BANKer R.D., Charnes A., CoOper W.W., Some models for estimating technical and scale inefficiencies in data envelopment analysis, Manage. Sci., 1984, 30 (9), 1078-1092. 
[3] COOPER W.W., SEIford L., TONE K., Introduction to data envelopment analysis and its uses, with DEA-Solver software and references, Springer, New York 2005.

[4] FÄre R., SvenSSON L., Congestion of production factors, Econ. J. Econ. Soc., 1980, 48, 1745-1753.

[5] FÄRE R., GROSSKOPF S., Measuring congestion in production, J. Econ., 1983, 43, 257-271.

[6] FÄre R., GrosskopF S., Lovell C.A.K., The measurement of efficiency of production, Kluwer, Boston 1985.

[7] CoOper W.W., ThOmpson R.G., Thrall R.M., Introduction extensions and new developments in DEA, Ann. Oper. Res., 1996, 66, 3-45.

[8] Brockett P.L., CoOper W.W., Shin H.C., WANG Y., Inefficiency and congestion in Chinese production before and after the 1978 economic reforms, Socio-Econ. Plan. Sci., 1998, 32, 1-20.

[9] Cooper W.W., Deng H., HuAng Z.M., Li S.L., A one model approach to congestion in DEA, Socio-Econ. Plan. Sci., 2002, 36, 231-238.

[10] JahanSHAHLOO G.R., KHODABAKHSHI M., Suitable combination of inputs for improving outputs in DEA with determining input congestion considering textile industry of China, Appl. Math. Comp., 2004, 151, 263-273.

[11] KHODABAKHSHI M., Chance constrained additive input relaxation model in stochastic data envelopment analysis, J. Inf. Syst. Sci., 2010, 6 (1), 99-112.

[12] WeI Q.L., YAN H., Congestion and returns to scale in data envelopment analysis, Eur. J. Oper. Res., 2004, 153, 641-660.

[13] Tone K., SAHOo B.K., Degree of scale economics and congestion, a unified DEA approach, Eur. J. Oper. Res., 2004, 153, 641-660.

[14] Sueyoshi T., SeKitiani K., DEA congestion and returns to scale under an occurrence of multiple optimal projections, Eur. J. Oper. Res., 2009, 194, 592-607.

[15] Mehdiloozad M., ZhU J., SAHOo B.K., Identification of congestion in data envelopment analysis under the occurrence of multiple projections. A reliable method capable of dealing with negative data, Eur. J. Oper. Res., 2018, 265, 644-654.

[16] Shadab M., SaAti S., FARzipoor Saen R., Mostafaee A., Measuring congestion by anchor points in $D E A$, J. Sadhana, 2019, DOI:10.1007/s12046-020-1274-y.

[17] Ebrahimzade Adimi M., Rostamy-MalKhalifeh M., HosseinZadeh Lotfi F., MeHrJoo R., A new linear to find the congestion hyperplane in DEA, J. Math. Sci., 2019, 13, 43-52.

[18] KeRSTENS K., VAN DE WOESTYNE I., The remarkable incidence of congestion in production. A review. Empirical illustration, and research agenda. Data Env. Anal. J., 2019, 4 (2), 109-147.

[19] Mostafaee A., SohraieE S., The role of hyperplanes for characterizing suspicious units in DEA, J. Ann. Oper. Res., 2018, 275 (2), 531-549. 